\title{
Grain Structure at Crack Path in Fatigued Nano-Crystalline Ni
}

\author{
Li-Chung Lai ${ }^{1,2}$, Wen-An Chiou ${ }^{1}$ and J.C. Earthman ${ }^{2}$ \\ 1. NISP Lab, Maryland NanoCenter, University of Maryland, College Park, MD 20742-2831 \\ 2. Dept. of Chemical Eng. and Materials Science, Univ. of California, Irvine, CA 92697-2575
}

The generally acceptable mechanisms of fatigued crack growth in nanocrystalline (NC) materials were based on empirical studies and molecular dynamics (MD) simulations of pure Ni films [1, 2]. MD simulation suggested that fatigued crack growth is due mainly to crack-tip blunting, nanovoid formation, subsequent decohesion at grain boundaries [2] while Meirom et al. suggested a crack propagation rate dependent and dislocation-slip mechanism [3]. However, these studies were based on two-dimensional thin films. The lack of 3-dimensional constraint and a high volume fraction of the surface, 2-D thin-film samples may not represent the mechanical behavior of bulk polycrystalline materials [4]. Furthermore, the investigations of fatigued crack structures in NC materials have been limited to surface studies using SEM; the lack of knowledge on the inner grain structure at the crack path impedes the further understanding of fatigued crack growth mechanism in NC materials. A crack propagation study of sufficient thick, full condensed 3-dimensional fatigued NC Ni with the emphasis of grain structure at crack path using TEM was thus undertaken.

Bulk NC Ni-99.9\% sheets $(0.5 \mathrm{~mm}$ in thickness with average grain size of $29 \mathrm{~nm})$ were fabricated using a pulsed electrodeposition technique (Integran Technologies Inc., Canada). Testing specimens $(37.5 \mathrm{~mm} \times 3.81 \mathrm{~mm} \times 0.5 \mathrm{~mm})$ were machined from the as-deposited sheets by electrical discharged machining (EDM) (Misubishi DWC90C with $0.25 \mathrm{~mm}$ brass wire), followed by mechanical polishing along the longitudinal direction to flatten the surface (final thickness $=0.43$ $\mathrm{mm}$ ) and remove transverse scratches that may serve as crack precursors. Tests were conducted with a three-point bend fatigue system under deflection-controlled conditions. After examining the crack/fracture surfaces with a SEM, crack/fracture surfaces were protected by electrodepositing a thick Ni layer to prevent possible artifacts before/during preparing TEM specimens.

TEM investigation, as shown in Figs. 1 though 5, clearly depicts deformation-induced grain growth along the crack path, which asserts Yang's finding [5]. The grown grain morphology is dependent on the stress intensity factor range $(\Delta K=C \Delta \sigma \sqrt{ } \pi a$ for opening crack mode, where $C$ is a geometry-related value, $\Delta \sigma=\sigma_{\max }-\sigma_{\min }$ is stress range, $a$ is crack length) which is associated with crack growth velocity [1]. At low $\Delta K$, the noticeable grain growth zone is about one or two grains in depth from crack path (Fig. 2). As $\Delta K$ increases (Figs. 2 to 4 or Fig.5a to b), grain growth zone may reach at least a few microns in depth which is hundreds of times the average grain size (though TEM observation is limited by the size of electron transparent area). The increase of $\Delta K$ also leads to the change in crack behavior. The crack propagation behavior changed from intergranular at low crack velocity ( $\Delta K=13$, indicated by less or equal to average grain size, Fig. 6 ) to partially transgranular at high crack velocity $(\Delta K=21$, indicated by large than average grain size, Fig. 7). Non-equilibrium grain boundaries (Fig. 7, curved arrows) resulting from grain growth activity may account for the facet cracks observed at crack tip advance direction. Twin crystals were believed to be a product of crack propagation in NC grains formed by Shockley partial dislocations due to stress. The absence of twins in the high $\Delta K$ region where noticeable grain growth occurred indicated that the crack behavior has changed. Thus, the mechanism of crack propagation is crack growth velocity/ $\Delta K$ dependent and related to grain growth mechanism.

[1] T. Hanlon et al., International Journal of Fatigue 27 (2005) 1147-1158.

[2] D. Farkas et al., Phys. Rev. Lett. 94 (2005) 165502.

[3] R.A. Meirom et al., Phys. Rev. Lett. 101 (2008) 085503.

[4] E. Ma, Scripta Mater. 49 (2003) 663-668.

[5] Y. Yang et al., Metall. and Mater. Trans. A 39A (2008) 1145-1156.

[6] This research was supported by NSF Grant D-DMR-0304629. TEM/SEM investigation was supported and performed in the NISP Lab, NanoCenter and MRSEC at University of Maryland. 


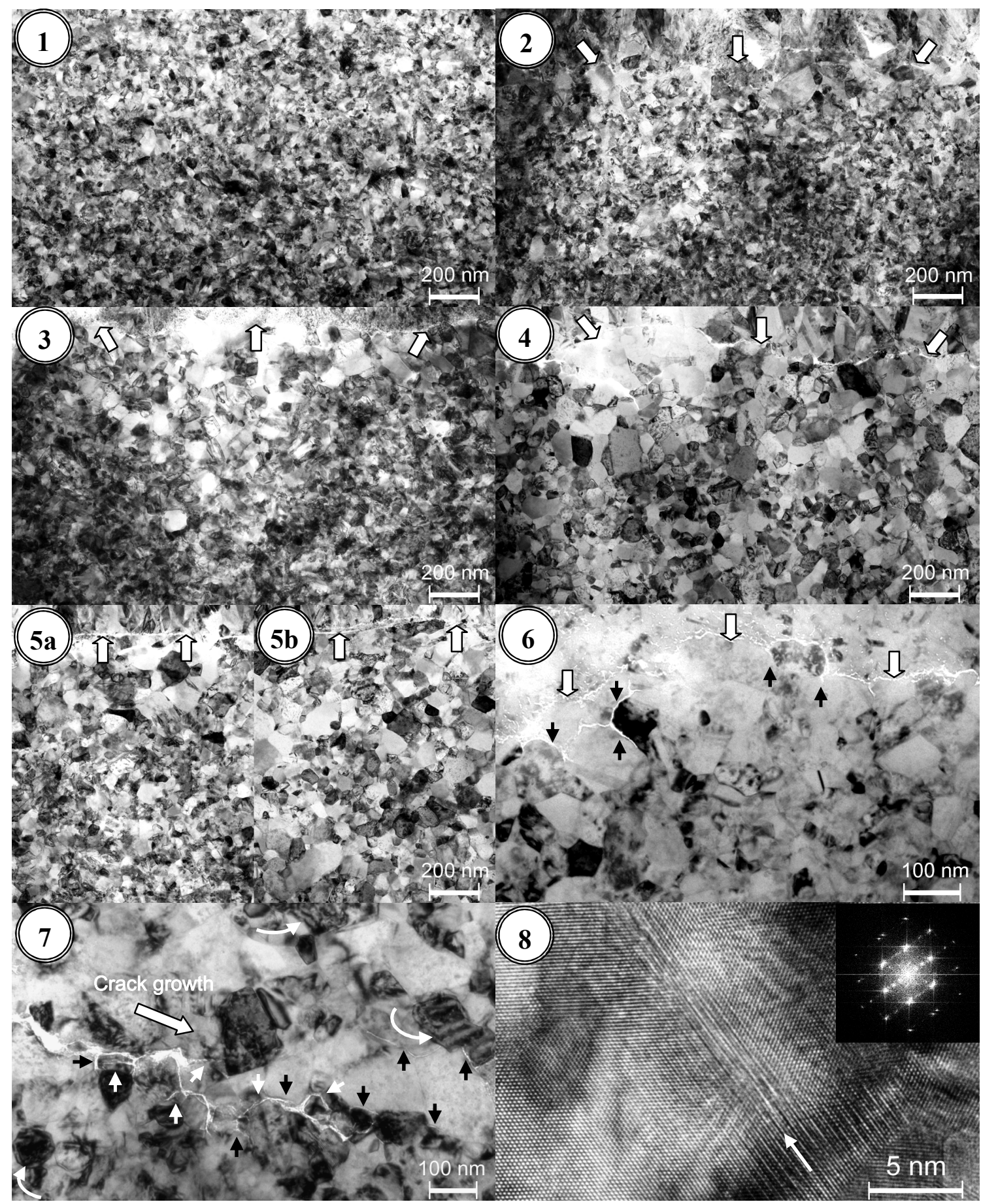

Fig. 1. STEM image of an as-received pulse electron-deposited Ni sheet showing nano-grain morphology.

Figs. 2 to 4. STEM images of fatigued samples at various stress range. STEM images were taken at the same distance $(\sim 100 \mu \mathrm{m})$ from crack initiation site. $\Delta K$ at each image was $\sim 6.5,13$, and 20 , respectively. The crack paths, i.e., fracture surfaces (arrows) are right below the deposited Ni protection layers.

Fig. 5. STEM images showing grain growth in the same TEM foil: (a) $\sim 20 \mu \mathrm{m}(\Delta K \sim 10)$, and (b) $\sim 100 \mu \mathrm{m}$ $(\Delta K \sim 20)$ away from crack initiation site. The crack paths are indicated by open arrows.

Fig. 6. TEM image of crack path (open arrows, $\Delta K \sim 13$ ) revealing intergranular cracks (dark arrows).

Fig. 7. TEM image of crack tip area $(120 \mu \mathrm{m}$ from the crack initiation site, $\Delta K \sim 21)$ showing transgranular (white arrows) and intergranular (black arrows) cracks with non-equilibrium gain boundary (curved arrow).

Fig. 8. High resolution image of crystal of twins found at crack path where $\Delta K \sim 6.5$. Inset: FFT pattern. 